\title{
Thyroid Warthin-Like Cancer Concurrent With Multiple Sclerosis: A Case Report
}

\author{
Lucia Stella Curto $^{\mathrm{a}, \mathrm{d}}$, Rita Gervasi ${ }^{\mathrm{b}}$, Valeria Zuccala ${ }^{\mathrm{c}}$, Nadia Innaro ${ }^{\mathrm{b}}$
}

\begin{abstract}
Warthin like-papillary thyroid cancer (WL-PTC) is a rare malignancy; it is difficult to distinguish preoperatively a WLPTC from a classic PTC. Often it is associated with Hashimoto thyroiditis (HT) that determines a better prognosis with a very low probability of recurrence. The case concerns a 43-year-old female with a single thyroid nodule suspected for cancer; and she had multiple sclerosis (MS) from the age of 19. Thyroid hormone levels were normal such as thyroid antibodies. Total thyroidectomy with lymphadenectomy of central compartment was performed. Histological examination revealed a Warthin like-PTC without Hashimoto thyroiditis. The mechanisms involved in pathogenesis of thyroid cancer in patients with autoimmune disease are not completely clear. We hypothesized that not only a local autoimmune response, such as HT, could contribute to the determination of this type of cancer but also a systemic autoimmune disease such as MS.
\end{abstract}

Keywords: Thyroid Warthin-like carcinoma; Autoimmune disease; Multiple sclerosis; Hashimoto thyroiditis

\section{Introduction}

Thyroid cancer (TC) is the most common endocrine malignancy. Among differentiated TCs, the papillary variety (PTC) is more frequently diagnosed. The last edition of World Health Organization (WHO) classification of thyroid tumors considers different variants such as papillary microcarcinoma, encapsulated, follicular, diffuse sclerosing, tall cell, columnar cell, cribriform-morular, hobnail (micro papillary/discohesive), fibromatosis/ fasciitis-like stroma, solid/trabecular, oncocytic, spindle cell, clear cell and Warthin-like [1].

Manuscript submitted November 24, 2020, accepted December 8, 2020

Published online December 30, 2020

aUnit of Clinical Surgery, Department of Medical and Surgical Sciences, Magna Graecia University Medical School, Catanzaro, Italy

${ }^{b}$ Unit of Endocrine Surgery, A.O.U. Mater Domini, Catanzaro, Italy

"Pathology Unit, "Pugliese Ciaccio" Hospital, Catanzaro, Italy

${ }^{\mathrm{d} C}$ Corresponding Author: Lucia Stella Curto, Unit of Clinical Surgery, Department of Medical and Surgical Sciences, Magna Graecia University Medical School, Catanzaro, Italy. Email: luciastella15@gmail.com
In 1995 Apel described for the first time thyroid Warthinlike cancer (WLPTC). This name comes from the near morphological similarity to the major salivary glands cancer. This variety is characterized by papillary architecture, oxyphil cytology and lymphocytic infiltrates in papillary stalks. He recognized a strength association with Hashimoto thyroiditis (HT) [2]. It seems that patients with WLPTC had similar demographic, clinical, pathologic, and molecular characteristics to those with classic PTC coexisting with HT [3]. Generally, its prognosis is similar to classical papillary cancer, but when associated with HT it seems to be less aggressive. The role of autoimmune systemic disease is still under evaluation; some studies reveal a relationship between thyroid disease and multiple sclerosis (MS) [4-7].

We report a singular case of thyroid Warthin-like carcinoma in a patient with MS, but without HT. Although it is clear the association with autoimmune thyroiditis, we want to underlines probably strength relationship with systemic autoimmune disease even without a local autoimmune thyroiditis.

\section{Case Report}

\section{Investigations}

A 43-year-old woman was admitted to the the Department of Endocrine Surgery of A.O.U. Mater Domini in Catanzaro for a cancer suspected thyroid single nodule. When she was 19 , she was diagnosed with MS.

\section{Diagnosis}

During the follow-up for this disease, in 2019, she was subjected to a complete thyroidal evaluation. The ecographic sonography revealed the presence of single nodule of $10 \times 11 \times 10 \mathrm{~mm}$ with hyperechogenic spots, in upper sinister lobe of the thyroid gland. No lymphadenomegaly was found. On February 2020, she underwent fine needle aspiration cytology (FNAC) evaluation. It was positive for TIR4 according to SIAPEC classification [8]. Thyroidal hormone and antibody dosage was normal.

\section{Treatment}

The patient underwent total thyroidectomy and biopsy of two 


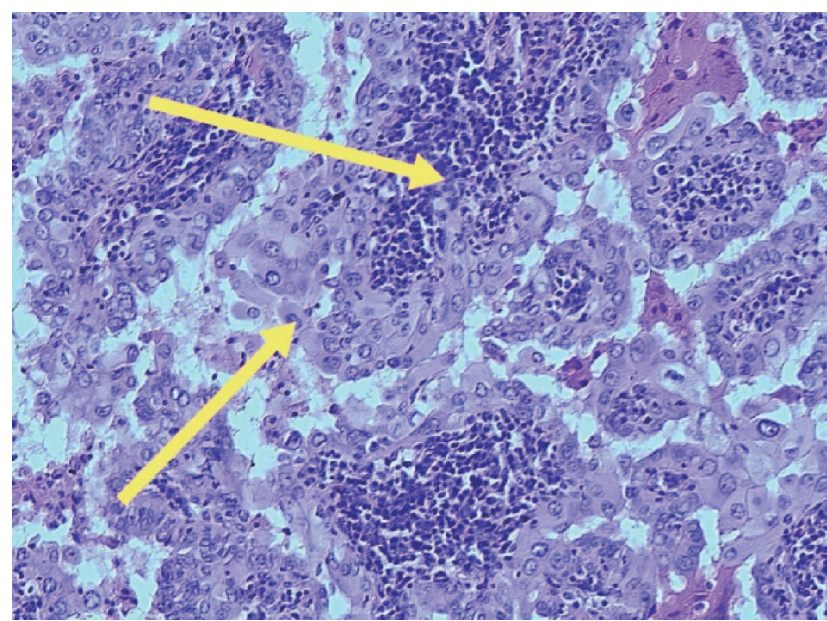

Figure 1. Cytological characteristic of WLPTC $(10 \times)$. The tumor cells lining the papillae are eosinophilic (lower arrow) and large, the cores of the papillae contain a prominent lymphoplasmacytic infiltrate (upper arrow). This variant shares histological features with Warthin tumor of salivary gland origin. WLPTC: Warthin like-papillary thyroid cancer.

lymph nodes of central compartment. IOMN was used during surgical procedure.

Postoperative period was uneventful and the patient was discharged on the second postoperative day. Calcium levels were always normal and patient never had dysphonia.

Pathological examination was compatible with papillary carcinoma Warthin-like variety; all lymph nodes were cancerfree. Dexter lobe was $3.5 \times 2 \times 1.5 \mathrm{~mm}$; sinister lobe was 4 $\times 2 \times 2.5 \mathrm{~mm}$. Dexter lobe had parenchymatous texture and appearance. Sinister lobe compounds a nodule within margins, white color, and size of $0.8 \mathrm{~cm}$. Cytological characteristic was as follow: papillary architecture, oxyphilic cytoplasm and stroma with dense lymphoplasma cellular infiltrate (Fig. 1).

However, there was no evidence of any extra-thyroidal extension or any nodal metastasis.

\section{Follow-up and outcomes}

After 7 months, no signs of local recurrence or metastases were observed.

\section{Discussion}

Warthin-like PTC is a rare entity; there was described only about 160 cases in the world. It is more common in women and median age of diagnosis is 47 years [9].

There are no specific sonographic characteristics or preoperative cytological patterns that could recognize earlier this type of cancer. However, surgical strategy is the same of a classic PTC. Only definitive pathology and immunochemistry can differentiate a PTC from a WLPTC. Often, changes seen in Warthin-like PTC are associated to autoimmune thyroiditis (88\%) [10]; its presence is considered a favorable prognostic factor. WLPTC in AT has lower rates of lymph node metasta- sis, extra-thyroidal extension and recurrence [11].

The lymphocytic infiltration may suggest a not well-defined role for immunological mechanisms in the pathogenesis of thyroid tumors. According to different study, Hashimoto's disease and MS are autoimmune disorders that may share similar mechanisms for the pathogenesis of the dysregulation of their self-immunity [4].

TCs have been described in immunosuppressed patient, especially with autoimmune disease [5]. The role of systemic autoimmune disease in the pathogenesis of TC was partially studied. There is a strength connection between systemic lupus erythematosus (SLE) and TC, even if the molecular mechanisms are not totally defined [6].

Therefore, we hypothesized a role of MS in the pathogenesis of WLPTC regardless of HT coexistence.

The mechanism involved is unclear; probably immunosuppressive treatments play an important role in association with Epstein-Barr virus (EBV) infection [7].

This case report is characteristic for its presentation: in our case, WLPTC is not associated with HT but with MS. So probably not only a local autoimmune response could contribute to the determination of this type of cancer but also a systemic autoimmune disease. Due to limited literature on this topic, our evaluation is based only on few studies, so further studies are needed to explain the molecular mechanism involved.

\section{Acknowledgments}

None to declare.

\section{Financial Disclosure}

None to declare.

\section{Conflict of Interest}

None to declare.

\section{Informed Consent}

The patient has provided permission to publish these features of her case and the identity of the patient has been protected.

\section{Author Contributions}

NI, VZ and LSC contributed to think up the design of manuscript. RG and LSC contributed to literature research and language revision. All authors wrote the manuscript.

\section{Data Availability}

The data described during the current study are available from 
the corresponding author on reasonable request.

\section{References}

1. Kakudo K, Bychkov A, Bai Y, Li Y, Liu Z, Jung CK. The new 4th edition World Health Organization classification for thyroid tumors, Asian perspectives. Pathol Int. 2018;68(12):641-664.

2. Apel RL, Asa SL, LiVolsi VA. Papillary Hurthle cell carcinoma with lymphocytic stroma. "Warthin-like tumor" of the thyroid. Am J Surg Pathol. 1995;19(7):810-814.

3. Yeo MK, Bae JS, Lee S, Kim MH, Lim DJ, Lee YS, Jung CK. The Warthin-like variant of papillary thyroid carcinoma: a comparison with classic type in the patients with coexisting Hashimoto's thyroiditis. Int J Endocrinol. 2015;2015:456027.

4. Sloka JS, Phillips PW, Stefanelli M, Joyce C. Co-occurrence of autoimmune thyroid disease in a multiple sclerosis cohort. J Autoimmune Dis. 2005;2:9.

5. Milosevic Z, Tanic N, Bankovic J, Stankovic T, Buta M, Lavrnic D, Milovanovic Z, et al. Genetic alterations in quadruple malignancies of a patient with multiple sclerosis: their role in malignancy development and response to therapy. Int J Clin Exp Pathol. 2014;7(4):1826-1833.

6. Zhang M, Li XM, Wang GS, Qian L, Tao JH, Ma Y, Li XP.
Thyroid cancer in systemic lupus erythematosus: a meta analysis. Int J Clin Exp Pathol. 2014;7(9):6270-6273.

7. Sirbu AM, Sirbu CA, Eftimie L, Soare AM, Ghinescu MC, Ionita-Radu F. Multiple sclerosis, human herpesvirus 4 and thyroid collision tumor: A case report. Exp Ther Med. 2020;20(4):3458-3461.

8. Nardi F, Basolo F, Crescenzi A, Fadda G, Frasoldati A, Orlandi F, Palombini L, et al. Italian consensus for the classification and reporting of thyroid cytology. J Endocrinol Invest. 2014;37(6):593-599.

9. Kim EY, Lee KH, Park YL, Park CH, Kim Dae-Hyun, Chae SW, Yun JS. Warthin-like papillary thyroid carcinoma accompanying classical papillary carcinoma: Report of three cases and systematic review of the literature. International Journal of Clinical and Experimental Pathology. 2017;10:7223-7231.

10. Paker I, Kokenek TD, Yilmazer D, Seker GE, Alper M. Oncocytic variant of papillary thyroid carcinoma with lymphocytic stroma (Warthin-like variant): report of a case with fine needle aspiration cytology and review of the literature. Cytopathology. 2012;23(6):408-410.

11. Gonzalez-Colunga KJ, Loya-Solis A, Cecenas-Falcon LA, Barboza-Quintana O, Rodriguez-Gutierrez R. Warthin-like papillary thyroid carcinoma associated with lymphadenopathy and Hashimoto's thyroiditis. Case Rep Endocrinol. 2015;2015:251898. 